\title{
A Criterion for Dynamic Ductile Fracture Initiation of Tensile Mode
}

\author{
Fuzuli Ağrı Akçay, ${ }^{1, *}$ Erkan Oterkus ${ }^{2}$
}

1 İstanbul Teknik Üniversitesi Gemi İnşaatı ve Deniz Bilimleri Fakültesi, Maslak, İstanbul 34469, Turkey.

2 Department of Naval Architecture, Ocean and Marine Engineering, University of Strathclyde, Glasgow G4 0LZ, UK.

*Corresponding author: Fuzuli Ağrı Akçay: akcayfu@itu.edu.tr

\begin{abstract}
Dynamic behavior of a material is essential in applications such as collision, explosion, ballistic impact, high speed machining, and metal forming. As impact loadings, as well as accidental or malicious explosions may impose high loading rates to engineering structures, estimating the dynamic response of a material accurately is crucial. Therefore, an analytical strain rate dependent criterion on ductile fracture initiation is developed at the continuum scale by further developing the energy balance concept. The criterion is based on continuum modeling of energy release rates and the critical state is reached when the rate of energy change of fractured and unfractured states become equal. The formulation introduces a material length scale and a material property that is a function of strain rate and temperature. The developed ductile fracture criterion is implemented into two example applications, an aluminum alloy and a titanium alloy, whose experimental data are obtained from the open literature. Fracture loci of these alloys at various strain rates and the critical energy release rates as a function of strain rate are determined. The results of the example applications agree well with the experimental results reported in the literature.
\end{abstract}

Keywords: Fracture; Ductility; Strain rate dependent; Plasticity; Energy release rate 


\section{Nomenclature}

\begin{tabular}{|c|c|}
\hline$A$ & area of the fracture plane \\
\hline$A_{1}, A_{2}, A_{3}$ & material constants \\
\hline$c_{V}$ & specific heat capacity at constant volume \\
\hline $\mathrm{C}_{I}$ & specific surface energy density \\
\hline$d u$ & plastic work increment per unit volume \\
\hline$d k$ & kinetic energy increment per unit volume \\
\hline$d w$ & mechanical work increment per unit volume (of the unfractured medium) \\
\hline$d W$ & mechanical work increment of the unfractured medium \\
\hline$d W^{*}$ & mechanical work increment of the fractured medium \\
\hline$d \varepsilon_{i j}$ & components of (plastic) strain increment tensor (of the unfractured medium) \\
\hline $\begin{array}{l}d \varepsilon_{I}, d \varepsilon_{I I}, d \varepsilon_{I I I} \\
d \varepsilon_{I}^{*}, d \varepsilon_{I I}^{*}, d \varepsilon_{I I I}^{*} \\
d \varepsilon_{e f f}\end{array}$ & $\begin{array}{l}\text { strain increments of the unfractured medium in principal directions } \\
\text { strain increments of the fractured medium in principal directions } \\
\text { equivalent (plastic) strain increment }\end{array}$ \\
\hline$l_{I, 0}$ & characteristic length (relevant to ductile fracture) \\
\hline$l_{I}, l_{I I}, l_{I I I}$ & current dimensions of the volume element \\
\hline$m, n$ & material constants \\
\hline$T$ & actual material temperature \\
\hline$T_{\text {melt }}$ & melting temperature of the material \\
\hline$T_{\text {room }}$ & room temperature \\
\hline$v_{I}, v_{I I}, v_{I I I}$ & components of velocity vector (in principal directions) \\
\hline$x_{I}, x_{I I}, x_{I I I}$ & principal directions \\
\hline$\beta$ & fraction of the plastic work contributing to temperature increase \\
\hline$\varepsilon_{i j}$ & components of true strain \\
\hline$\dot{\varepsilon_{0}}$ & reference strain rate \\
\hline$\dot{\varepsilon}_{I}, \dot{\varepsilon_{I I}}, \dot{\varepsilon_{I I I}}$ & true strain rates in principal directions \\
\hline$\varepsilon_{e f f}$ & equivalent (plastic) strain \\
\hline$\overline{\varepsilon_{f}}$ & equivalent (plastic) strain at fracture \\
\hline$\lambda$ & non-negative scalar factor \\
\hline$\rho$ & mass density of the material \\
\hline$\sigma_{i j}$ & components of true stress tensor \\
\hline$\sigma_{I}, \sigma_{I I}, \sigma_{I I I}$ & principal stresses of the unfractured medium \\
\hline$\sigma_{I}^{*}, \sigma_{I I}^{*}, \sigma_{I I I}^{*}$ & principal stresses of the fractured medium \\
\hline$\sigma_{e f f}$ & equivalent stress \\
\hline$\Gamma_{I}$ & critical effective energy release rate (of Mode I fracture) \\
\hline$\Delta T$ & increase in temperature \\
\hline
\end{tabular}




\section{Introduction}

Impact loadings, as well as accidental or malicious explosions may impose high loading rates to engineering structures (Grimsmo et al., 2015). Therefore, dynamic behavior of a material, which can significantly differ from its static one (Avriel et al., 2018), is essential in applications such as collision, explosion, ballistic impact, high speed machining, metal forming, etc. As the choice of the constitutive model and the identification of model parameters are vital (Özel \& Karpat, 2007), a large number of research studies that investigated the dynamic response of materials focused on establishing a plastic constitutive model. Plastic constitutive models can be divided into two categories: Microstructure based physical models and empirical phenomenological models (Khan \& Liu, 2012b). In addition, plastic constitutive models can be categorized as rate independent models or rate dependent models. Interested readers on rate independent constitutive models are referred to recent articles by Siddiq (2019), Asim et al. (2020), and Fu et al. (2020). On the other hand, rate dependent constitutive models are discussed more in detail in the following paragraphs.

Zerilli \& Armstrong (1987) model is an example of microstructure based physical model. The model is based on dislocation mechanics and the constitutive equations were developed for body-centered-cubic (BCC) and face-centered-cubic (FCC) materials. Later, Voyiadjis \& Abed (2005b) discussed the derivation of Zerilli-Armstrong constitutive equations and they proposed a model that accounts for the evolution of mobile dislocation density. Various models that consider the effect of dislocation density evolution on the response of different crystal structures are available in the literature (see e.g., Voyiadjis \& Abed, 2005a; Rusinek \& RodríguezMartínez, 2009; Austin \& McDowell, 2011; Gao \& Zhang, 2012; Khan et al., 2015; Shahba \& Ghosh, 2016). A widespread use of microstructure based physical models is limited, as they generally involve a large number of parameters (Siddiq \& Schmauder, 2005).

An example of empirical phenomenological model is Johnson \& Cook (1983) model. Johnson \& Cook (1983) presented a constitutive model that primarily aims to ease numerical implementation. The model includes five material constants, and the authors provided the numerical values of these constants for twelve different materials. Another example of empirical phenomenological model was proposed by Khan \& Liang (1999) based on the experimental results of three BCC metals (tantalum, tantalum alloy with $2.5 \%$ tungsten, and AerMet 100 steel). This model has been subjected to several modifications based on the observations on the mechanical responses of polycrystalline materials during nearly two 
decades (Khan \& Liu, 2012b; Kabirian et al., 2014). In addition to those mentioned, numerous phenomenological models have been proposed in the literature (see e.g., Molinari \& Ravichandran, 2005; Ulacia et al., 2011; Shojaei et al., 2013). Interested readers are referred to Sung et al. (2010), Huh et al. (2014) and Tanimura et al. (2014), where a summary and comparison of several strain rate (and temperature) dependent constitutive models are presented.

Material failure models can be divided into physically based models and empirical phenomenological models as well. Additionally, they can be divided into two categories as coupled models and uncoupled models. The former approach couples the constitutive model and the fracture criterion, that is, damage accumulation is incorporated into the constitutive model. A well-known example of this class is the Gurson model (Gurson, 1977). Gurson model, developed for porous ductile materials, is based on the void growth mechanics and has been subjected to modifications to account for realistic values (see e.g., Tvergaard, 1981) as well as shear dominated stress states (see, e.g., Nahshon \& Hutchinson, 2008 for shear modification and Nielsen \& Tvergaard, 2010 for further extension). Interested readers are referred to Benzerga et al. (2016) for a comprehensive review of Gurson based models. Moreover, variational methods have been utilized as well in order to model damage accumulation (see e.g., Siddiq et al., 2012; Placidi et al., 2018a; 2018b; 2019). On the other hand, the latter approach decouples damage accumulation and the constitutive model, that is, fracture criterion and the constitutive model act independent from each other. Hancock-Mackenzie model (Hancock \& Mackenzie, 1976) can be given as an example for this class.

In contrast to the plastic constitutive models, only a small portion of studies (that investigated the dynamic response of materials) focused on establishing a ductile fracture criterion for dynamic loading conditions. More than three decades ago, Johnson \& Cook (1985) proposed a strain rate (and temperature) dependent fracture criterion based on the results of a series of tensile tests on three different metals (OFHC copper, Armco iron, and 4340 steel). Although the original version is still used in the literature, Johnson-Cook fracture model has been exposed to modifications as well (see e.g., Børvik et al., 2001). Other strain rate dependent ductile fracture criteria have been proposed in addition to Johnson-Cook based failure criteria. Kim et al. (2008) proposed two failure criteria (using the effective strain or damage parameters) as a function of Zener-Holloman parameter (Zener \& Hollomon, 1944) to predict the fracture behavior of magnesium alloy sheets. The proposed criteria are based on observations and deep drawing experiments of AZ31 sheets were conducted in order to validate the proposed criteria. 
Khan \& Liu (2012c) proposed an empirical strain rate (and temperature) dependent fracture criterion, based on the magnitude of stress vector criterion (Khan \& Liu, 2012a), and the criterion was calibrated for Al2024-T351 alloy. Liu \& Sun (2014) proposed a dynamic ductile fracture model that involves the effect of hydrostratic pressure, Lode angle and the strain rate. The criterion was applied to determine the material parameters of Al-7075 alloy. Roth \& Mohr (2014) proposed an empirical extension of the Hosford-Coulomb model (Mohr \& Marcadet, 2015) to include the strain rate effect on fracture initiation. The expression was calibrated and validated using the experimental results of DP590 steel and TRIP780 steel. Although a comprehensive review on dynamic ductile fracture lacks, interested readers are referred to Molinari et al. (2014) for dynamic necking and fragmentation, and dynamic damage by microvoiding with applications to spalling and dynamic crack growth.

Currently, the original (Johnson \& Cook, 1985) or slightly modified versions (see e.g., Børvik et al., 2001) of Johnson-Cook fracture criterion is commonly used to account for the effect of strain rate (and temperature). However, Johnson-Cook fracture criterion and its modifications experience fundamental shortcomings as presented in the following. Firstly, Johnson-Cook criterion is based on stress triaxiality only; therefore, it cannot capture the difference between different stress states of same triaxiality. Nevertheless, experimental studies have shown that the Lode angle/parameter (that is, the third stress invariant) plays an important role (in addition to stress triaxiality) in ductile fracture process (see e.g., Wierzbicki et al., 2005; Barsoum \& Faleskog, 2007; Bai \& Wierzbicki, 2010; Charoensuk et al., 2017). Hence, both parameters need to be taken into account to predict ductile fracture accurately. Although researchers have modified Johnson-Cook criterion in order to include the Lode angle dependency, this results in extra material constant(s), which requires additional experiment(s) to calculate these new constant(s) (see e.g., Erice \& Gálvez, 2014). Secondly, Johnson-Cook fracture criterion exhibits mesh size dependency (Larsson et al., 2016). Thirdly, Johnson-Cook based fracture criteria as well as above-mentioned fracture criteria are empirical, and an analytical strain rate dependent ductile fracture criterion still lacks. Therefore, the aim of the current study is to develop an analytical strain rate dependent criterion on ductile fracture initiation at the continuum scale. This relationship is obtained by further developing the energy balance concept (Karr \& Akçay, 2016). The energy-based criterion to be developed includes the stress triaxiality and the Lode angle dependencies inherently, without the necessity of extra material constants, by considering the stress state as a whole. Moreover, as the criterion to be developed introduces a characteristic length, use of characteristic length as a mesh size removes mesh dependency. The novelty of 
the current research is development of a rate dependent ductile fracture criterion (of tensile mode) that is physically based, mesh independent and encompasses stress triaxiality and Lode angle dependencies inherently.

Dynamic loading here and thereafter corresponds to non-cylic loading cases, that is, fatigue loading is beyond the scope of the current investigation and studied elsewhere (see e.g., Andreaus \& Baragatti, 2012; Andreaus et al., 2017; Nguyen et al., 2020; Liao et al., 2020). The article is organized as follows. The ductile fracture criterion is derived in Section 2, and implemented into two example applications in Section 3. The results are presented and discussed in Section 4. Finally, a summary with conclusion remarks are given in Section 5.

\section{Ductile Fracture Criterion for Dynamic Loading}

In this section, an analytical strain rate dependent criterion on ductile fracture initiation at the continuum scale is to be developed. This relationship is obtained by further developing the energy balance concept (Karr \& Akçay, 2016) and the focus is given only tensile mode (i.e., Mode I) fracture.

Conservation of energy principle requires the sum of the rate of work done on a body by external forces and all other energy rates being equal to the time rate of change of the internal energy and the kinetic energy (Eringen, 1980). In the following, the case where the work done on the system is only by external forces is considered, that is, no other external energy enters the system. It is also assumed that there are no pre-existing cracks or damage at the continuum scale. Moreover, elastic deformation (i.e., elastic strain energy) can be ignored as ductile fracture usually associated with large strains where plastic deformation dominates. The mechanical work increment per unit volume, $d w$, then takes the form of

$$
d w=d u+d k=\sigma_{i j} d \varepsilon_{i j}+\rho v_{i} d v_{i}
$$

where $d u$ and $d k$ represent the plastic work increment and the kinetic energy increment per unit volume, respectively. Also, $\sigma_{i j}$ are the components of true stress tensor, $d \varepsilon_{i j}$ are the components of (plastic) strain increment tensor, $\rho$ is the mass density of the material, and $v_{i}$ are the components of velocity vector.

Let consider an isotropic continuous medium in a rectangular cartesian coordinate system subjected to uniform state of stress before fracture. Suppose that this medium has the current 
dimensions of $l_{I}, l_{I I}$, and $l_{I I I}$ in the principal directions of $x_{I}, x_{I I}$, and $x_{I I I I}$, respectively. The volume element with a potential fracture plane is presented in Figure 1.

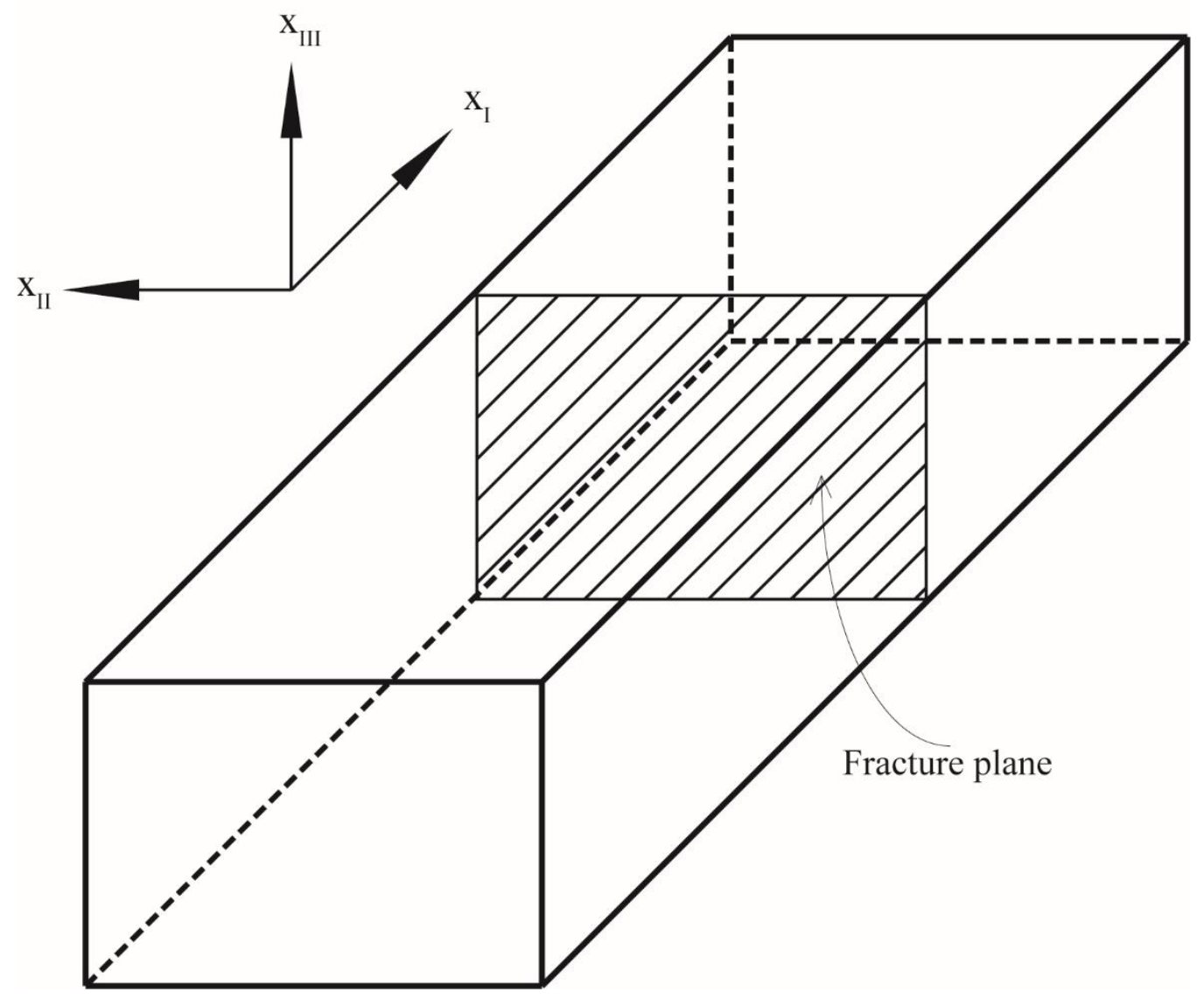

Figure 1 Volume element and potential fracture plane

The mechanical work increment per unit volume of the unfractured medium can be written in terms of principal values as

$$
d w=\sigma_{I} d \varepsilon_{I}+\sigma_{I I} d \varepsilon_{I I}+\sigma_{I I I} d \varepsilon_{I I I}+\rho\left(v_{I} d v_{I}+v_{I I} d v_{I I}+v_{I I I} d v_{I I I}\right)
$$

where $\sigma_{I} \geq \sigma_{I I} \geq \sigma_{I I I}$. Also, the natural strain components in the principal directions are defined as

$$
d \varepsilon_{I}=d l_{I} / l_{I}, d \varepsilon_{I I}=d l_{I I} / l_{I I}, d \varepsilon_{I I I}=d l_{I I I} / l_{I I I}
$$

Integrating Eq. (3) yields

$$
\varepsilon_{I}=\ln \frac{l_{I}}{l_{I, 0}}, \quad \varepsilon_{I I}=\ln \frac{l_{I I}}{l_{I I, 0}}, \quad \varepsilon_{I I I}=\ln \frac{l_{I I I}}{l_{I I I, 0}}
$$


where $l_{i, 0}$ and $l_{i}$ are the initial and the current length components of the volume element, respectively. Now, let take the time derivative of Eq. (4)

$$
\dot{\varepsilon}_{I}=\frac{1}{l_{I}} \frac{d l_{I}}{d t}, \quad \dot{\varepsilon}_{I I}=\frac{1}{l_{I I}} \frac{d l_{I I}}{d t}, \quad \varepsilon_{I I I}^{\cdot}=\frac{1}{l_{I I I}} \frac{d l_{I I I}}{d t}
$$

where $\dot{\varepsilon}_{I}, \dot{\varepsilon}_{I I}, \varepsilon_{I I I}$ are the true strain rates in the principal directions.

Rigid body translational or rotational velocities are not considered (in the calculation of kinetic energy increment) as they do not make any contribution to fracture process. In other words, the 'effective/active' velocity is $v_{i}=d l_{i} / d t$ not $v_{i}=d x_{i} / d t$, with $x_{i}$ representing the components of position vector. Hence, the velocity components can simply be written as

$$
v_{I}=l_{I} \dot{\varepsilon}_{I}, v_{I I}=l_{I I} \dot{\varepsilon}_{I I}, v_{I I I}=l_{I I I} \varepsilon_{I I I}^{\cdot}
$$

Eq. (6) is in accordance with the true strain rate definition given by Dieter (1986). Differentiating both sides of Eq. (6) yields

$$
\begin{gathered}
d v_{I}=l_{I} d \dot{\varepsilon}_{I}+\dot{\varepsilon}_{I} d l_{I} \\
d v_{I I}=l_{I I} d \dot{\varepsilon_{I I}}+\dot{\varepsilon_{I I}} d l_{I I} \\
d v_{I I I}=l_{I I I} d \dot{\varepsilon_{I I I}}+\dot{\varepsilon}_{I I}^{\cdot} d l_{I I I}
\end{gathered}
$$

It is presumed that the strain rate remains nearly constant during an increment, that is, $d \dot{\varepsilon}_{I}=$ $d \varepsilon_{I I}=d \varepsilon_{I I I}^{\cdot}=0$. Hence, Eq. (7) reduces to

$$
d v_{I}=\dot{\varepsilon}_{I} d l_{I}, d v_{I I}=\varepsilon_{I I} d l_{I I}, d v_{I I I}=\varepsilon_{I I I}^{:} d l_{I I I}
$$

Substituting Eq. (3) into Eq. (8) yields

$$
d v_{I}=l_{I} \dot{\varepsilon}_{I} d \varepsilon_{I}, d v_{I I}=l_{I I} \dot{\varepsilon}_{I I} d \varepsilon_{I I}, d v_{I I I}=l_{I I I} \varepsilon_{I I I} d \varepsilon_{I I I}
$$

Finally, substituting Eqs. (6) and (9) into Eq. (2), and multiplying by the current volume yield the mechanical work increment of the unfractured medium, $d W$

$$
\begin{gathered}
d W=l_{I} l_{I I} l_{I I I}\left\{\left[\sigma_{I}+\rho\left(l_{I} \dot{\varepsilon}_{I}\right)^{2}\right] d \varepsilon_{I}+\left[\sigma_{I I}+\rho\left(l_{I I} \dot{\varepsilon_{I I}}\right)^{2}\right] d \varepsilon_{I I}\right. \\
\left.+\left[\sigma_{I I I}+\rho\left(l_{I I I} \varepsilon_{I I I}\right)^{2}\right] d \varepsilon_{I I I}\right\}
\end{gathered}
$$

This expression, i.e., Eq. (10), provides the necessary amount of energy increment for the system to deform without fracture; that is, $d E$. On the other hand, the necessary amount of 
energy increment for the system to fracture, $d E^{*}$, includes an additional energy that is required to create new surfaces (Karr \& Akçay, 2016), that is

$$
d E^{*}=\left(\Gamma_{I} \times A\right) d \varepsilon_{I}+d W^{*}
$$

where

$$
\begin{gathered}
d W^{*}=l_{I} l_{I I} l_{I I I}\left\{\left[\sigma_{I}^{*}+\rho\left(l_{I} \dot{\varepsilon}_{I}^{*}\right)^{2}\right] d \varepsilon_{I}^{*}+\left[\sigma_{I I}^{*}+\rho\left(l_{I I} \dot{\varepsilon}_{I I}^{*}\right)^{2}\right] d \varepsilon_{I I}^{*}\right. \\
\left.+\left[\sigma_{I I I}^{*}+\rho\left(l_{I I I} \varepsilon_{I I I}^{*}\right)^{2}\right] d \varepsilon_{I I I}^{*}\right\}
\end{gathered}
$$

Here $\Gamma_{I}$ denotes the critical effective energy release rate per unit surface area of the fracture plane (of Mode I fracture) and $d W^{*}$ is the mechanical work increment of the fractured medium. Mode I fracture plane and its area, which is $A=l_{I I} l_{I I I}$, are shown in Figure 1. Also, all stress, strain, and strain rate quantities denoted by star are associated with the fractured medium. Moreover, the critical effective energy release rate $(\Gamma)$ is a material property for a particular fracture mode (Karr \& Akçay, 2016), and it can be a function of strain rate and temperature.

The rate of energy change of the unfractured medium is initially less than the fractured medium, as the system maintains the minimum energy state. In other words, the system, seeking the minimum energy state, will fracture if the necessary energy increment for the fractured state becomes less than the necessary energy increment for the unfractured state. Therefore, the critical state is reached when the rate of energy change of these two states becomes equal (Karr $\&$ Akçay, 2016). Hence, mathematical representation of the critical state is

$$
d E=d E^{*}
$$

with principal stresses and strains, and strain rates maintained consistent with the boundary conditions, that is

$$
\begin{gathered}
\sigma_{I I}^{*} d \varepsilon_{I I}^{*}=\sigma_{I I} d \varepsilon_{I I},\left(\dot{\varepsilon_{I I}^{*}}\right)^{2} d \varepsilon_{I I}^{*}=\left(\dot{\varepsilon_{I I}}\right)^{2} d \varepsilon_{I I} \\
\sigma_{I I}^{*} d \varepsilon_{I I I}^{*}=\sigma_{I I I} d \varepsilon_{I I I},\left(\dot{\varepsilon_{I I I}^{*}}\right)^{2} d \varepsilon_{I I I}^{*}=\left(\varepsilon_{I I I}^{*}\right)^{2} d \varepsilon_{I I I}
\end{gathered}
$$

As the increment in strain is concentrated in the fracture zone, $d \varepsilon_{I}^{*}$ suddenly drops to zero; hence, the first term (in the paranthesis) of Eq. (12) vanishes. Therefore, the critical state condition, i.e., Eq. (13), takes the following form 


$$
l_{I} l_{I I} l_{I I I}\left[\sigma_{I}+\rho\left(l_{I} \dot{\varepsilon}_{I}\right)^{2}\right] d \varepsilon_{I}=\left(\Gamma_{I} \times A\right) d \varepsilon_{I}
$$

Substituting $A=l_{I I} l_{I I I}$ into Eq. (15) and simplifying it yields

$$
l_{I}\left[\sigma_{I}+\rho\left(l_{I} \dot{\varepsilon}_{I}\right)^{2}\right]=\Gamma_{I}
$$

Now, let define

$$
C_{I}=\frac{\Gamma_{I}}{l_{I, 0}}
$$

Finally, substituting Eqs. (4) and (17) into Eq. (16) yields the critical state for Mode I fracture

$$
e^{\varepsilon_{I}}\left[\sigma_{I}+\rho\left(l_{I} \dot{\varepsilon}_{I}\right)^{2}\right]=\mathrm{C}_{I}
$$

As presented above, $l_{I, 0}$ and $l_{I}$ are the initial and the current length of the volume element, respectively, in the maximum principal stress/strain direction. Use of a material feature (e.g., a characteristic length) for $l_{I, 0}$ such that the stress state within the volume element remains uniform at the continuum scale is suggested. Hence, in this case, $C_{I}$ is a material property, and can be a function of strain rate and temperature. Physically, $\mathrm{C}_{I}$ represents the specific surface energy density as it refers to critical energy release rate per unit (characteristic) length (Karr \& Akçay, 2016).

The critical state condition given by Eq. (18) does not include any terms that take the history of state of stress or strain into consideration. Hence, this form of the equation is suggested to be utilized for proportional loading conditions. On the other hand, a slight improvement to Eq. (18) that can be used for non-proportional loading cases is proposed in the following

$$
e^{\int_{0}^{\varepsilon_{e q}^{f}}\left(1 / \zeta_{I}\right) d \varepsilon_{e q}}\left[\sigma_{I}+\rho\left(l_{I} \dot{\varepsilon}_{I}\right)^{2}\right]=\mathrm{C}_{I}
$$

where

$$
\begin{aligned}
\zeta_{I} & =\sqrt{\frac{2}{3}\left[1+\left(\frac{d \varepsilon_{I I}}{d \varepsilon_{I}}\right)^{2}+\left(\frac{d \varepsilon_{I I I}}{d \varepsilon_{I}}\right)^{2}\right]} \\
d \varepsilon_{e q} & =\sqrt{\frac{2}{3}\left[\left(d \varepsilon_{I}\right)^{2}+\left(d \varepsilon_{I I}\right)^{2}+\left(d \varepsilon_{I I I}\right)^{2}\right]}
\end{aligned}
$$


Here, plastic incompressibility is assumed and $\zeta_{I}$ is assumed to remain constant during an increment. $d \varepsilon_{e q}$ denotes the equivalent (plastic) strain increment with $\varepsilon_{e q}$ denoting the equivalent (plastic) strain. The integral term in Eq. (19) takes the deformation history into account; hence, use of Eq. (19) is suggested for non-proportional loading cases. Accordingly, use of the integral term $\int_{0}^{\varepsilon_{e q}^{f}}\left(1 / \zeta_{I}\right) d \varepsilon_{e q}$ is suggested for non-proportional loading cases (instead of equivalent plastic strain itself $\left.\left(\varepsilon_{e q}\right)\right)$ in the graphical illustration of fracture locus. If the loading is proportional, $\zeta_{I}$ remains constant throughout loading and Eq. (19) reduces to Eq. (18).

There is not sufficient time for appreciable heat flow to occur at high rates of deformation (Dieter, 1986) due to sudden and abrupt nature of the crack nucleation process (Carlioz et al., 2020). Therefore, in high loading cases, where the plastic work cannot be totally dissipated, there will be temperature increase in the material. Although a temperature term does not explicitly appear in the critical state equation (Eq. (19)), the effect of temperature increase should be included implicitly by determining the current temperature after each increment, and updating the values of temperature-dependent terms, particularly $\sigma_{I}$ (i.e., the constitutive equations) and $C_{I}$, accordingly. The increase in temperature, $\Delta T$, can be computed by

$$
\Delta T=\frac{\beta}{\rho c_{V}} \int \sigma_{i j} d \varepsilon_{i j}
$$

where $\beta$ is the fraction of the plastic work contributing to temperature increase, and $c_{V}$ is the specific heat capacity at constant volume. Here, the thermal energy term is considered to be only generated by mechanical work; however, extraneous heat sources may exist in the system that are not purely mechanical (see e.g., Rittel, 2014 for details). Additionally, the reason for the use of the specific heat capacity at constant volume is that the plastic flow is essentially isochoric (Kapoor \& Nemat-Nasser, 1998).

\section{Example Applications}

Numerous experimental studies have been conducted in order to explore the effect of dynamic loading on fracture behavior of metals, including various type of steels (Børvik et al., 2005; Lee et al., 2007; Børvik et al., 2009; Amini \& Nemat-Nasser, 2010; Erice et al., 2012; Kim et al., 2013; Anderson et al., 2014; Vaz-Romero et al., 2015; Majzoobi et al., 2016; Grimsmo et al., 2017; Chiyatan \& Uthaisangsuk, 2020), aluminium alloys (Clausen et al., 2004; Smerd et al., 
2005; El-Magd \& Abouridouane, 2006; Chen et al., 2009; Bobbili et al., 2016; Pan et al., 2016), magnesium alloys (El-Magd \& Abouridouane, 2006; Yu et al., 2017), titanium alloys (El-Magd \& Abouridouane, 2006; Zheng et al., 2015; Bobbili \& Madhu, 2016; Huang et al., 2018; Wang et al., 2019), nickel alloys (Sjöberg et al., 2017), tungten alloys (Rittel \& Weisbrod, 2001), and tantalum (Dorogoy \& Rittel, 2017). In addition, fracture behavior of additively manufactured metals is a rising topic of research interest (see e.g., Spagnuolo et al., 2017; De Angelo et al., 2019; Yu et al., 2019) thanks to rapid development of 3D-printing technology. However, in this section, the experimental results of Bobbili et al. (2016) and Bobbili \& Madhu (2016) are to be utilized, as the articles were published recently and the authors provided enough data of interest (i.e., true fracture strain values for various stress states and strain rates) to investigate the application of the proposed criterion.

The von-Mises equivalent stress in terms of principal stresses is defined as

$$
\sigma_{e q}=\sqrt{\frac{1}{2}\left[\left(\sigma_{I}-\sigma_{I I}\right)^{2}+\left(\sigma_{I I}-\sigma_{I I I}\right)^{2}+\left(\sigma_{I I I}-\sigma_{I}\right)^{2}\right]}
$$

Also, for a given stress state, it is convenient to express the equivalent stress in terms of maximum principal stress, that is

$$
\sigma_{e q}=\xi_{I} \sigma_{I}
$$

with $\xi_{I}$ takes the following form for the axisymmetric stress state (Karr \& Akçay, 2016)

$$
\xi_{I}=\frac{3}{2+3 \eta}
$$

where $\eta$ is the stress triaxiality.

Moreover, the flow rule is expressed as (Desai \& Siriwardane, 1984)

$$
\begin{aligned}
& d \varepsilon_{I}=\frac{2}{3} \lambda\left[\sigma_{I}-\frac{1}{2}\left(\sigma_{I I}+\sigma_{I I I}\right)\right] \\
& d \varepsilon_{I I}=\frac{2}{3} \lambda\left[\sigma_{I I}-\frac{1}{2}\left(\sigma_{I}+\sigma_{I I}\right)\right] \\
& d \varepsilon_{I I I}=\frac{2}{3} \lambda\left[\sigma_{I I I}-\frac{1}{2}\left(\sigma_{I}+\sigma_{I I}\right)\right]
\end{aligned}
$$

where $\lambda$ is a non-negative scalar factor and may vary throughout loading. 
Bobbili et al. (2016) and Bobbili \& Madhu (2016) conducted tensile tests on Al-4.8Cu-1.2Mg alloy and $\mathrm{Ti}-10 \mathrm{~V}-2 \mathrm{Fe}-3 \mathrm{Al}$ alloy to investigate the fracture behavior of these alloys at differrent loading rates. They provided experimental data for four different stress triaxialities at four strain rates. However, as there was no explicit information on the fracture mode of the smooth specimens, only the data of notched specimens (i.e., three data points for each strain rate) are considered here, that is, $\eta=0.5153, \eta=0.6209$, and $\eta=0.7387$. Moreover, the authors did not provide any data for strain rate beyond $1000 \mathrm{~s}^{-1}$ for $\mathrm{Al}-4.8 \mathrm{Cu}-1.2 \mathrm{Mg}$ alloy and $1500 \mathrm{~s}^{-1}$ for Ti-10V-2Fe-3Al alloy. Hence, Johnson-Cook fracture criterion (Johnson \& Cook, 1985) is used to calculate the approximate fracture strains at any loading case beyond these strain rates. The fracture strain values at these loading cases are calculated for the same three stress triaxialities. Furthermore, although proportional loading is not generally the case in actual experiments, it is assumed that the stress triaxiality remains nearly constant throughout loading, as the authors did not provide any experimental data on the evolution of stress triaxiality. Hence, for the proportional loading case, as mentioned above, $\zeta_{I}$ remains constant throughout loading with $\zeta_{I}=1$ for the axisymmetric case (Karr \& Akçay, 2016).

Johnson-Cook constitutive model (Johnson-Cook, 1983) is used to represent the constitutive behavior of $\mathrm{Al}-4.8 \mathrm{Cu}-1.2 \mathrm{Mg}$ and $\mathrm{Ti}-10 \mathrm{~V}-2 \mathrm{Fe}-3 \mathrm{Al}$ alloys, as Bobbili et al. (2016) and Bobbili \& Madhu (2016) used Johnson-Cook constitutive model as well, and they provided the material constants of the model. The authors also proposed a slightly modified version of Johnson-Cook constitutive equation to improve the prediction accuracy of the flow behavior of the materials of interest at high strain rates and temperatures (Bobbili et al., 2016; Bobbili \& Madhu, 2016). However, the original Johnson-Cook constitutive equation, given in the following, is used here as it describes the flow behavior of the materials of interest fairly well, as well.

$$
\sigma_{e q}=\left[A_{1}+A_{2} \varepsilon_{e q}^{n}\right]\left[1+A_{3} \ln \left(\frac{\dot{\varepsilon}}{\dot{\varepsilon_{0}}}\right)\right]\left[1-\left(\frac{T-T_{\text {room }}}{T_{\text {melt }}-T_{\text {room }}}\right)^{m}\right]
$$

where $\sigma_{e q}, \varepsilon_{e q}, \dot{\varepsilon}$, and $\dot{\varepsilon_{0}}$ are the equivalent stress, equivalent plastic strain, strain rate, and reference strain rate, respectively. Also, $T, T_{\text {room }}$, and $T_{\text {melt }}$ are the actual material temperature, room temperature and the melting temperature of the material, respectively. Moreover, there are five material constants $\left(A_{1}, A_{2}, A_{3}, n, m\right)$ to be determined. Different strategies on the calculation of these material parameters were extensively discussed by Gambirasio \& Rizzi (2014) and the material constants were determined for three materials (i.e., DH36 structural 
steel, a commercially pure niobium and an AL-6XN stainless steel) using the published experimental data.

\subsection{Example 1: Mode I Fracture of Al-4.8Cu-1.2Mg}

Bobbili et al. (2016) conducted tensile tests on Al-4.8Cu-1.2Mg alloy at four different strain rates: $0.01 \mathrm{~s}^{-1}, 0.1 \mathrm{~s}^{-1}, 1 \mathrm{~s}^{-1}, 1000 \mathrm{~s}^{-1}$. Nevertheless, the specific surface energy density, $\mathrm{C}_{I}$, is determined for three additional strain rates as well; i.e., for $5000 \mathrm{~s}^{-1}, 20000 \mathrm{~s}^{-1}, 50000 \mathrm{~s}^{-1}$. The approximate fracture strains at these strain rates are calculated by using Johnson-Cook fracture criterion, given by Bobbili et al. (2016) as

$$
\overline{\varepsilon_{f}}=\left[0.05+1.281 e^{-3.5 \eta}\right]\left[1+0.138 \ln \left(\frac{\dot{\varepsilon}}{\dot{\varepsilon_{0}}}\right)\right]
$$

Again, as mentioned previously, Eq. (28) is used to determine the fracture strains only for three stress triaxialities in order to obtain the approximate value of $\mathrm{C}_{I}$ at strain rates beyond $1000 \mathrm{~s}^{-}$ 1 .

Moreover, the following constitutive equation, whose material constants are provided by Bobbili et al. (2016), is used to predict the flow behavior of $\mathrm{Al}-4.8 \mathrm{Cu}-1.2 \mathrm{Mg}$ alloy

$$
\sigma_{e f f}=\left[380+482 \varepsilon_{e f f}^{0.51}\right]\left[1+0.0128 \ln \left(\frac{\dot{\varepsilon}}{\dot{\varepsilon_{0}}}\right)\right]
$$

with the reference strain rate is taken as $\dot{\varepsilon}_{0}=1 \mathrm{~s}^{-1}$.

There is no published data on mass density or average spacings of large inclusions of $\mathrm{Al}-4.8 \mathrm{Cu}-$ $1.2 \mathrm{Mg}$ alloy, to the authors' best knowledge. Additionally, although the temper designation of the $\mathrm{Al}$ alloy is provided as T6, its commercial name is not known (personal communication with Dr. Bobbili through e-mail). However, the copper and magnesium contents of Al-4.8Cu1.2Mg alloy remains within the chemical composition range of Al 2024 and Al 2124 alloys (Cayless RBC, 2010). Therefore, properties of these two alloys are considered to approximate the mass density and characteristic length of $\mathrm{Al}-4.8 \mathrm{Cu}-1.2 \mathrm{Mg}$ alloy.

Mass densities of Al 2024 and Al 2124 alloys are 2770 kg/m³ (ASM Handbook Committee, 2010); hence, the mass density of Al-4.8Cu-1.2Mg alloy is taken as $\rho=2770 \mathrm{~kg} / \mathrm{m}^{3}$. Even though center to center spacing of inclusions for Al 2024 and Al 2124 alloys with T6 temper condition are not provided in the open literature (to the authors' best knowledge), Hahn \& Rosenfield (1975) provided center to center spacing of inclusions for Al 2024 and Al 2124 
alloys with T851 temper condition. Hence, considering the average value of (center to center spacing of inclusions for) Al 2024-T851 and Al 2124-T851 alloys gives a characteristic length of $l_{I, 0} \approx 8 \mu \mathrm{m}$. Despite the fact that temper condition of the alloy of interest is different than those considered in the prediction of the characteristic length, $l_{I, 0} \approx 8 \mu \mathrm{m}$ is a reasonable approximation, as it is on the order of inclusion spacing of other 2xxx Al alloys as well, for instance, Al 2014-T6 alloy (see e.g., Hahn \& Rosenfield, 1975).

\subsection{Example 2: Mode I Fracture of Ti-10V-2Fe-3Al (Ti-1023)}

Bobbili \& Madhu (2016) conducted tensile tests on Ti-10V-2Fe-3Al (also called Ti-10-2-3) alloy at four different strain rates as well: $0.01 \mathrm{~s}^{-1}, 500 \mathrm{~s}^{-1}, 1000 \mathrm{~s}^{-1}, 1500 \mathrm{~s}^{-1}$. Again, the specific surface energy density, $\mathrm{C}_{I}$, is determined for three additional strain rates; i.e., for $5000 \mathrm{~s}^{-1}$, $20000 \mathrm{~s}^{-1}, 50000 \mathrm{~s}^{-1}$, and the approximate fracture strains at these strain rates are calculated by using Johnson-Cook fracture criterion. The expression was given by Bobbili \& Madhu (2016) as

$$
\overline{\varepsilon_{f}}=\left[-0.04+0.46 e^{0.21 \eta}\right]\left[1+0.027 \ln \left(\frac{\dot{\varepsilon}}{\dot{\varepsilon_{0}}}\right)\right]
$$

Again, Eq. (30) is used to determine the fracture strains only for three stress triaxialities in order to obtain the approximate value of $\mathrm{C}_{I}$ at strain rates beyond $1500 \mathrm{~s}^{-1}$.

In addition, the following constitutive equation, whose material constants are provided by Bobbili \& Madhu (2016), is used to predict the flow behavior of Ti-10V-2Fe-3Al alloy

$$
\sigma_{e f f}=\left[842+754 \varepsilon_{e f f}^{0.42}\right]\left[1+0.021 \ln \left(\frac{\dot{\varepsilon}}{\dot{\varepsilon_{0}}}\right)\right]
$$

with the reference strain rate is taken as $\dot{\varepsilon}_{0}=1 \mathrm{~s}^{-1}$.

Mass density of Ti-10V-2Fe-3Al alloy is $\rho=4650 \mathrm{~kg} / \mathrm{m}^{3}$ (Boyer et al., 1998). Moreover, the defect spacing (only inclusions and pores that participated the fracture process are included) of Ti-10V-2Fe-3Al alloy was provided in Figure 15 of Moody et al. (1993) for various samples that were subjected to different processing conditions. Taking an average value of these data gives a charactarestic length of $l_{I, 0} \approx 12 \mu \mathrm{m}$. 


\section{Results and Discussion}

The specific surface energy densities of $\mathrm{Al}$ and $\mathrm{Ti}$ alloys of interest for a wide range of strain rates are presented in Figure 2 and Figure 3, respectively. Red circles are determined using the crititical state equation (with actual experimental data or data obtained through Johnson-Cook fracture criterion) and a curve fit of red circles are presented as a blue solid line. The data used for the lowest four strain rates are the experimental data, whereas Johnson-Cook data are used for the remaining three strain rates. The equation of the curve fit (blue solid line) takes the form of $C_{I}=999.5+2.5 \times 10^{-7}\left[2.86+\log \left(\frac{\dot{\varepsilon}}{\dot{\varepsilon_{0}}}\right)\right]^{11.4}$ for the Al-4.8Cu-1.2Mg alloy (see Figure 2), and $C_{I}=1011+6.5 \times 10^{-7}\left[2.98+\log \left(\frac{\dot{\varepsilon}}{\varepsilon_{0}}\right)\right]^{11.6}$ for the Ti-10V-2Fe-3Al alloy (see Figure $3)$.

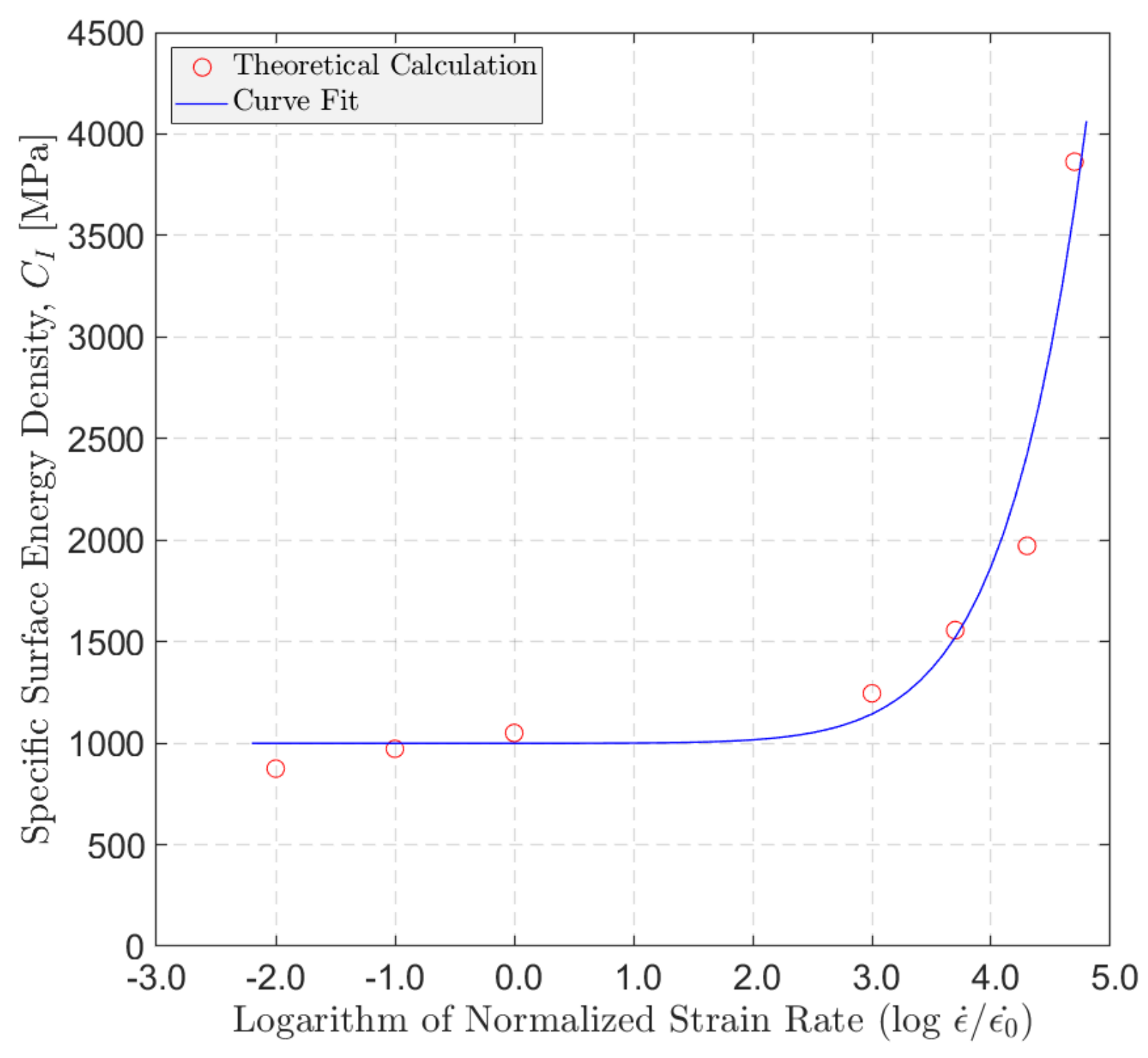

Figure 2 Specific surface energy density of $\mathrm{Al}-4.8 \mathrm{Cu}-1.2 \mathrm{Mg}$ alloy for various strain rates 


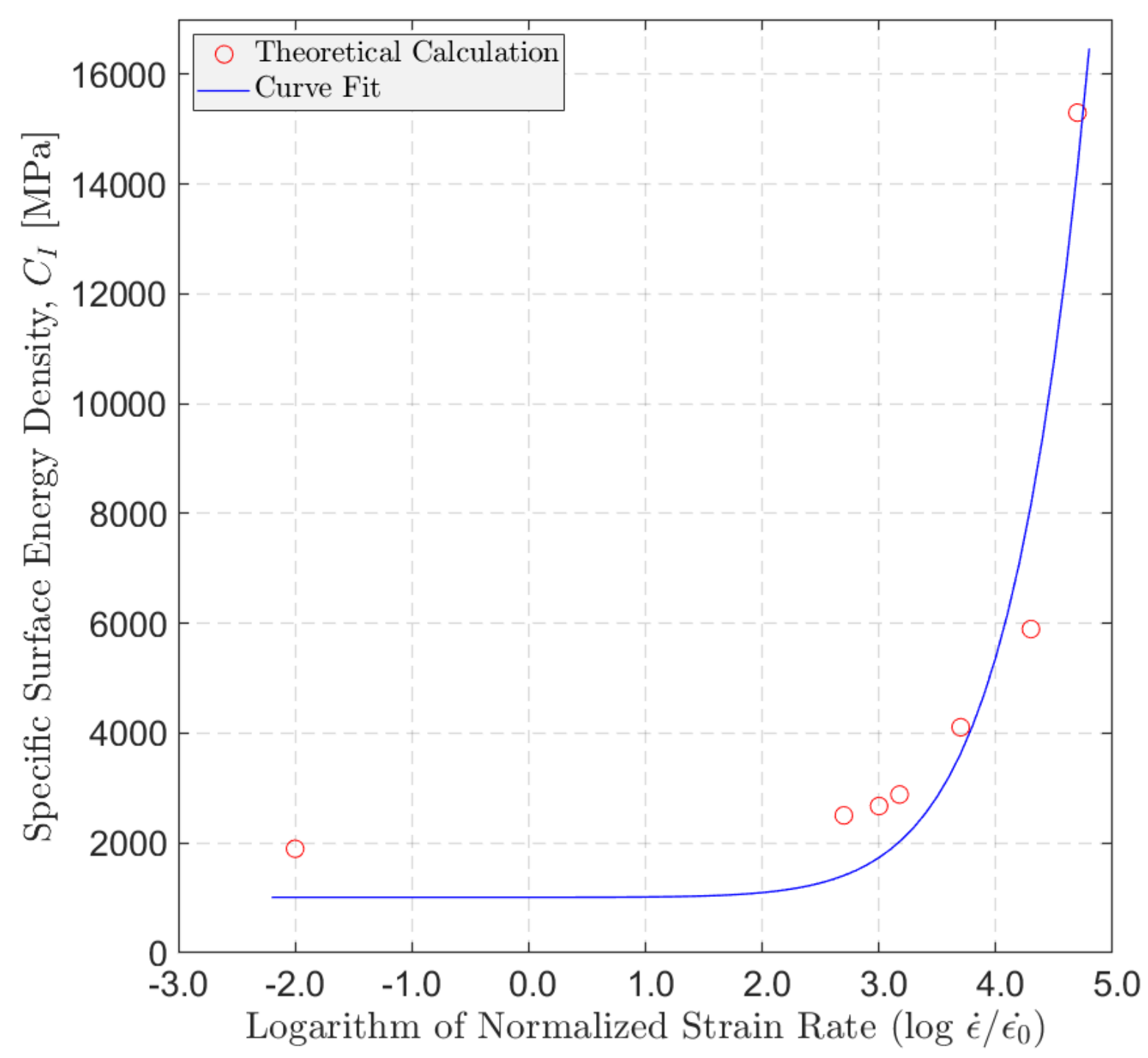

Figure 3 Specific surface energy density of Ti-10V-2Fe-3Al alloy for various strain rates

As can be seen from Figure 2 and Figure 3, the specific surface energy density (hence, the critical energy release rate) remains nearly constant at low strain rates. The specific surface energy density for the $\mathrm{Al}-4.8 \mathrm{Cu}-1.2 \mathrm{Mg}$ and $\mathrm{Ti}-10 \mathrm{~V}-2 \mathrm{Fe}-3 \mathrm{Al}$ alloys at low strain rates are determined as on the order of $C_{I}^{A l} \approx 1000 \mathrm{MPa}$ and $C_{I}^{T i} \approx 2000 \mathrm{MPa}$, respectively. These values correspond to critical energy release rates of $\Gamma_{I}^{A l} \approx 8 \mathrm{~kJ} / \mathrm{m}^{2}$ and $\Gamma_{I}^{T i} \approx 24 \mathrm{~kJ} / \mathrm{m}^{2}$ for Al4.8Cu-1.2Mg alloy and Ti-10V-2Fe-3Al alloy, respectively. The determined critical energy release rates are consistent with the energy release rates of $\mathrm{Al}$ and Ti alloys provided by Hahn et al. (1972).

On the other hand, the specific surface energy density (hence, the critical energy release rate) increases dramatically at high strain rates, that is, the specific surface energy density and the critical energy release rate are highly dependent on the loading rate at high strain rates. The 
trend of increase in critical energy release rate with strain rate, particularly at high strain rates, agrees with the results reported in the literature. Both experimental (see e.g., Owen et al., 1998) and numerical (see e.g., Osovski et al., 2015) studies showed that the effect of loading rate on energy release rate is little at small loading rates, whereas energy release rate is found to increase dramatically at high loading rates. Actually, the second term in Eqs. (18) and (19) explains the reason behind these observations. As can be seen from these equations, the second term is proportional to square of the strain rate and it doesn't contribute to the response very much until it approaches on the order of maximum principal stress $\left(\sigma_{I}\right)$. In other words, the specific surface energy density (hence, the critical energy release rate) does not change significantly until a critical strain rate (at which the second term approaches on the order of maximum principal strain) and then increases dramatically as the second term in the critical state equation is proportional to the square of the strain rate. Physically, material rate sensitivity and the material microstructure were found to be the main governers of this response (Osovski et al., 2015). The critical state equation is in accordance with this finding as well. In the equation, the maximum principal stress $\left(\sigma_{I}\right)$ represents the material rate sensitivity, whereas the material microstucture is represented by the characteristic length $\left(l_{I, 0}\right)$.

Fracture loci of $\mathrm{Al}$ and $\mathrm{Ti}$ alloys of interest for three differet strain rates are shown in Figure 4 and Figure 5, respectively. Fracture locus curves for $\dot{\varepsilon}=20000 \mathrm{~s}^{-1}$ is determined by evaluating the data obtained by Johnson-Cook fracture criterion, whereas the remaining two curves are obtained by evaluating experimental data presented by Bobbili et al. (2016) and Bobbili \& Madhu (2016). Newton-Raphson method is used to solve the critical state equation. 


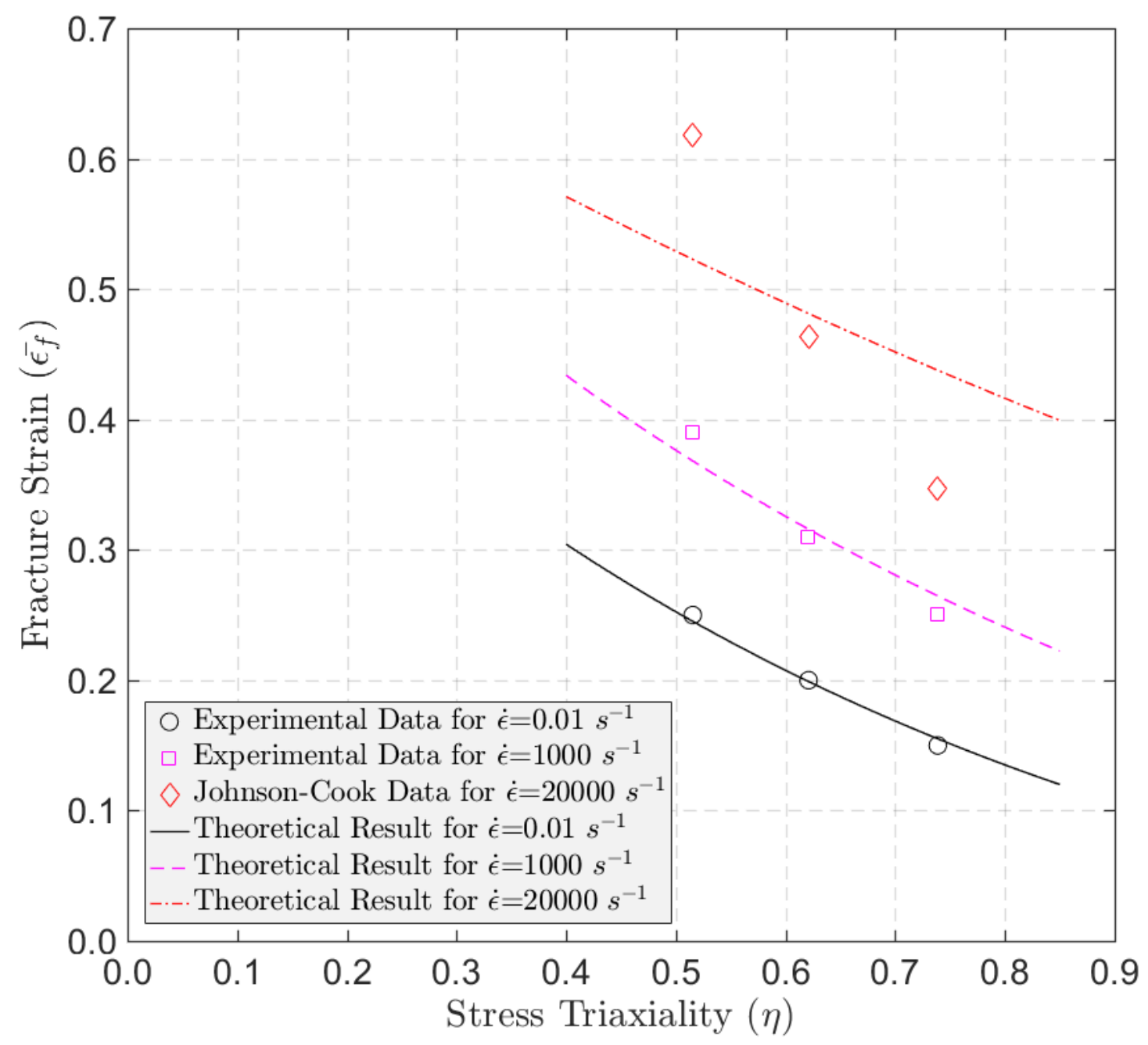

Figure 4 Fracture locus of $\mathrm{Al}-4.8 \mathrm{Cu}-1.2 \mathrm{Mg}$ alloy for various strain rates 


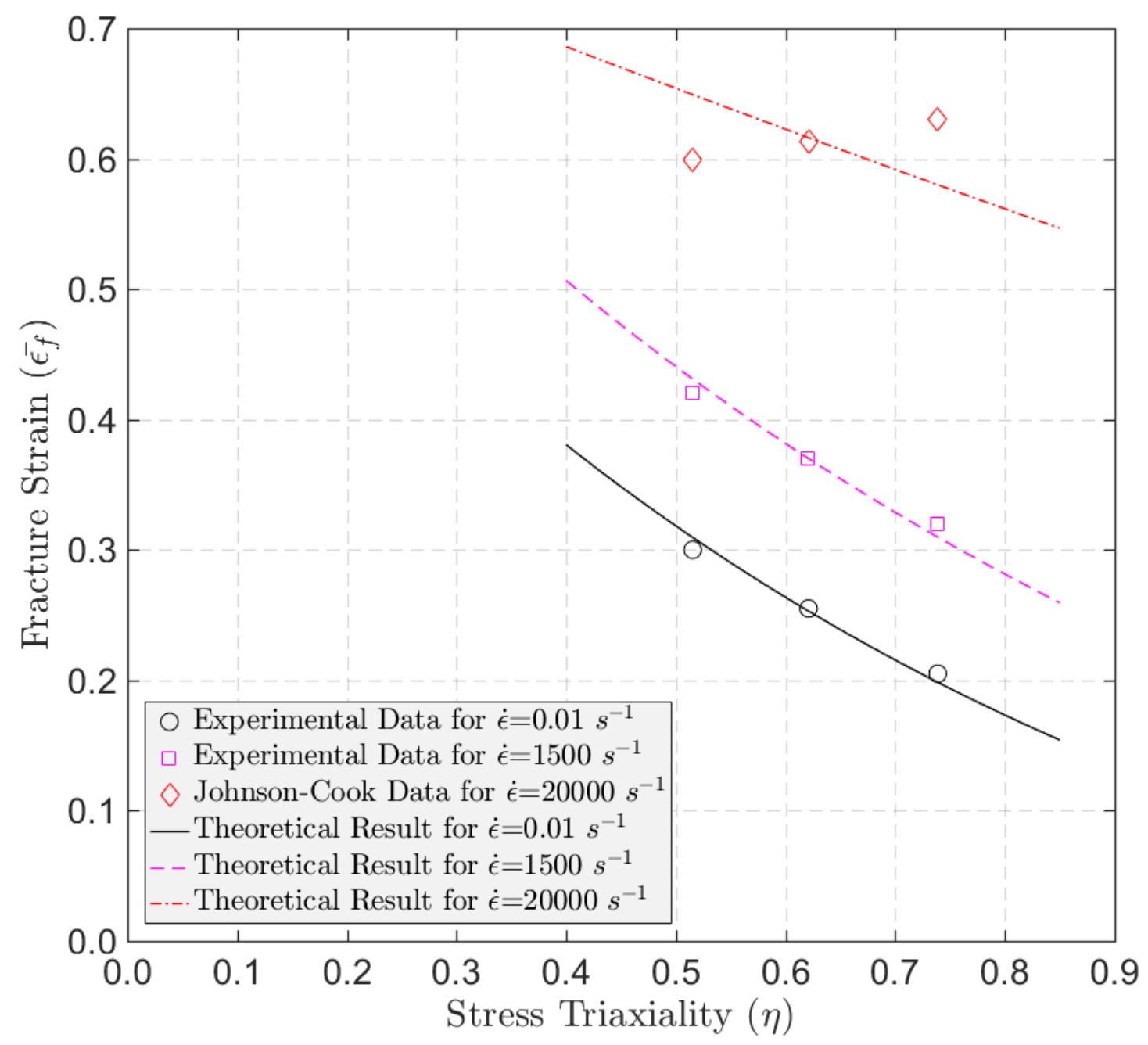

Figure 5 Fracture locus of Ti-10V-2Fe-3Al alloy for various strain rates

An interesting observation from Figure 4 and Figure 5 is that the fracture locus loses its nonlinear characteristic as the strain rate increases and it turns into nearly linear at ultra-high strain rates (the classification of strain rates in this article is based on the classification proposed by Piao et al., 2016) as can be seen from red dash-dot lines. However, determined fracture strains for the highest strain rate considered (i.e., $\dot{\varepsilon}=20000 \mathrm{~s}^{-1}$ ) do not agree well with the fracture strains estimated by Johnson-Cook criterion. The main reason for this disagreement is believed to be the fact that Johnson-Cook fracture criterion is an empirical criterion and may not produce accurate results for strain rates much higher than the strain rates used to determine the model parameters. Moreover, proportional loading assumption, neglecting temperature increase and the use of Johnson-Cook constitutive model are believed to slightly contribute to this disagreement as well.

As stated before, proportional loading is not generally the case in actual experiments, and the temperature of the material increases at high loading rates. Nevertheless, neither loading history 
nor temperature changes throughout loading are considered in the example applications presented here, as no data on the history of stress/strain state were provided by Bobbili et al. (2016) and Bobbili \& Madhu (2016). However, both loading history and temperature changes should be considered for the proper application of the critical state equation. The values of temperature-dependent terms, particularly $\sigma_{I}$ (i.e., the constitutive equations) and $C_{I}$, should be updated accordingly, in every increment. Moreover, the choice of constitutive model is crucial as well, as strain hardening, strain rate, and temperature sensitivity of the material needs to be accurately modeled for the appropriate and correct use of the developed fracture criterion.

Furthermore, as Bobbili et al. (2016) and Bobbili \& Madhu (2016) did not provide any data on local strain rates, global strain rates are used for the example applications considered. However, local strain rate can be different than global strain rate and it is location-dependent (Shahba \& Ghosh, 2016). Therefore, use of local strain rate is suggested for the appropriate application of the proposed criterion.

\section{Conclusions}

The energy balance concept developed by Karr \& Akçay (2016), which is based on continuum modeling of energy release rates, is further developed to include the effect of dynamic loading into consideration. The formulation introduces a material length scale $\left(l_{I, 0}\right)$ and a material property $\left(C_{I}\right)$ that is a function of strain rate and temperature. $\mathrm{C}_{I}$ is a material property only in the case of use of a material feature (e.g., a characteristic length) for $l_{I, 0}$ such that the stress state within the volume element remains uniform at the continuum scale. Physically, $\mathrm{C}_{I}$ represents the specific surface energy density as it refers to critical energy release rate per unit (characteristic) length (Karr \& Akçay, 2016).

The proposed ductile fracture criterion is implemented into two example applications, Al4.8Cu-1.2Mg alloy and $\mathrm{Ti}-10 \mathrm{~V}-2 \mathrm{Fe}-3 \mathrm{Al}$ alloy, whose experimental data were published by Bobbili et al. (2016) and Bobbili \& Madhu (2016). The fracture loci of these two alloys at various strain rates, and the specific surface energy densities as a function of strain rate are determined. The fracture locus is found to lose its non-linear characteristic as the strain rate increases, and it turns into nearly linear at ultra-high strain rates (see Piao et al., 2016 for the classification of strain rates). Moroever, the specific surface energy densities (hence, the critical energy release rates) of the considered alloys are found to be highly dependent on the loading rate, particulary at high strain rates. The trend of increase in critical energy release rate with strain rate, particularly at high strain strain rates, is in accordance with the results reported in 
the literature (see e.g., Owen et al., 1998; Osovski et al., 2015). Moroeveor, determined critical energy release rates of $\mathrm{Al}-4.8 \mathrm{Cu}-1.2 \mathrm{Mg}$ alloy and $\mathrm{Ti}-10 \mathrm{~V}-2 \mathrm{Fe}-3 \mathrm{Al}$ alloy are consistent with the energy release rates of $\mathrm{Al}$ and Ti alloys provided by Hahn et al. (1972).

On the other hand, the fracture criterion proposed in this article is applicable to tensile mode (i.e., Mode I) ductile fracture only. However, in practical applications, fracture may not be pure tensile mode; shear mode fracture, i.e., Mode II fracture (see e.g., Landau et al., 2016; Longère, 2018) as well as mixed mode fracture (see e.g., Longère \& Dragon, 2015) have been observed commonly at high strain rates. Therefore, development of a companion ductile fracture criterion of shear mode for dynamic loading conditions is vital and an avenue of continuing study.

\section{Acknowlegement}

The first author would like thank Dr. Osman Darıcı for his kind help to obtain some articles.

\section{Conflict of Interest}

The authors declare no conflict of interest.

\section{Funding}

This research did not receive any specific grant from funding agencies in the public, commercial, or not-for-profit sectors.

\section{References}

Amini MR, Nemat-Nasser S (2010). Micromechanisms of ductile fracturing of DH-36 steel plates under impulsive loads and influence of polyurea reinforcing. International Journal of Fracture, 162, 205-217.

Anderson D, Winkler S, Bardelcik A, Worswick MJ (2014). Influence of stress triaxiality and strain rate on the failure behavior of a dual-phase DP780 steel. Materials \& Design, 60, 198207.

Andreaus U, Baragatti P (2012). Experimental damage detection of cracked beams by using nonlinear characteristics of forced response. Mechanical Systems and Signal Processing, 31, 382-404.

Andreaus U, Baragatti P, Casini P, Iacoviello D (2017). Experimental damage evaluation of open and fatigue cracks of multi-cracked beams by using wavelet transform of static response via image analysis. Structural Control and Health Monitoring, 24, e1902.

Asim UB, Siddiq MA, McMeeking RM, Kartal ME (2020). A multiscale constitutive model for metal forming of dual phase titanium alloys by incorporating inherent deformation and failure mechanisms. arXiv preprint arXiv: 2002.04459. 
ASM Handbook Comittee (2010). Properties of wrought aluminum and aluminum alloys. In ASM Handbook, Volume 2: Properties and Selection: Nonferrous Alloys and Special-Purpose Materials, pp. 62-122. ASM International, Materials Park.

Austin RA, McDowell DL (2011). A dislocation-based constitutive model for viscoplastic deformation of fcc metals at very high strain rates. International Journal of Plasticity, 27, 1-24. Avriel E, Lovinger Z, Nemirovsky R, Rittel D (2018). Investigating the strength of materials at very high strain rates using electromagnetically driven expanding cylinders. Mechanics of Materials, 117, 165-180.

Bai Y, Wierzbicki T (2010). Application of extended Mohr-Coulomb criterion to ductile fracture. International Journal of Fracture, 161, 1-20.

Barsoum I, Faleskog J (2007). Rupture mechanisms in combined tension and shearExperiments. International Journal of Solids and Structures, 44, 1768-1786.

Benzerga AA, Leblond JB, Needleman A, Tvergaard V (2016). Ductile failure modeling. International Journal of Fracture, 201, 29-80.

Bobbili, R, Madhu V (2016). Effect of strain rate and stress triaxiality on tensile behavior of Titanium alloy Ti-10-2-3 at elevated temperatures. Materials Science and Engineering: A, 667, 33-41.

Bobbili R, Paman A, Madhu V (2016). High strain rate tensile behavior of Al-4.8Cu-1.2Mg alloy. Materials Science and Engineering: A, 651, 753-762.

Børvik T, Dey S, Clausen AH (2009). Perforation resistance of five different high-strength steel plates subjected to small-arms projectiles. International Journal of Impact Engineering, 36, 948964.

Børvik T, Hopperstad OS, Berstad T, Langseth M (2001). A computational model of viscoplasticity and ductile damage for impact and penetration. European Journal of MechanicsA/Solids, 20, 685-712.

Børvik T, Hopperstad OS, Dey S, Pizzinato EV, Langseth M, Albertini C (2005). Strength and ductility of Weldox $460 \mathrm{E}$ steel at high strain rates, elevated temperatures and various stress triaxialities. Engineering Fracture Mechanics, 72, 1071-1087.

Boyer R, Welsch G, Collings EW (1998). Materials properties handbook: Titanium alloys. ASM International, Materials Park.

Carlioz T, Dormieux L, Lemarchand E (2020). Thermodynamics of crack nucleation. Continuum Mechanics and Thermodynamics, 32, 1515-1531.

Cayless RBC (2010). Alloy and temper designation systems for aluminum and aluminum alloys. In ASM Handbook, Volume 2: Properties and Selection: Nonferrous Alloys and SpecialPurpose Materials, pp. 15-28. ASM International, Materials Park.

Charoensuk K, Panich S, Uthaisangsuk V (2017). Damage initiation and fracture loci for advanced high strength steel sheets taking into account anisotropic behaviour. Journal of Materials Processing Technology, 248, 218-235.

Chen Y, Clausen AH, Hopperstad OS, Langseth M (2009). Stress-strain behaviour of aluminium alloys at a wide range of strain rates. International Journal of Solids and Structures, 46, 3825-3835.

Clausen AH, Børvik T, Hopperstad OS, Benallal A (2004). Flow and fracture characteristics of aluminium alloy AA5083-H116 as function of strain rate, temperature and triaxiality. Materials Science and Engineering: A, 364, 260-272.

Chiyatan T, Uthaisangsuk V (2020). Mechanical and fracture behavior of high strength steels under high strain rate deformation: Experiments and modelling. Materials Science and Engineering: A, 779, 139125. 
De Angelo M, Spagnuolo M, D’Annibale F, Pfaff A, Hoschke K, Misra A, Dupuy, C, Peyre P, Dirrenberger J, Pawlikowski M (2019). The macroscopic behavior of pantographic sheets depends mainly on their microstructure: Experimental evidence and qualitative analysis of damage in metallic specimens. Continuum Mechanics and Thermodynamics, 31(4), 1181-1203.

Desai CS, Siriwardane HJ (1984). Constitutive laws for engineering materials with emphasis on geologic materials. Prentice-Hall, New Jersey.

Dieter GE (1986). Mechanical metallurgy. McGraw-Hill, New York.

Dorogoy A, Rittel D (2017). Dynamic large strain characterization of tantalum using shearcompression and shear-tension testing. Mechanics of Materials, 112, 143-153.

El-Magd E, Abouridouane M (2006). Characterization, modelling and simulation of deformation and fracture behaviour of the light-weight wrought alloys under high strain rate loading. International Journal of Impact Engineering, 32, 741-758.

Erice B, Gálvez F (2014). A coupled elastoplastic-damage constitutive model with Lode angle dependent failure criterion. International Journal of Solids and Structures, 51, 93-110.

Erice B, Gálvez F, Cendón DA, Sánchez-Gálvez V (2012). Flow and fracture behaviour of FV535 steel at different triaxialities, strain rates and temperatures. Engineering Fracture Mechanics, 79, 1-17.

Eringen AC (1980). Mechanics of continua. Robert E. Krieger Publishing Company, Melbourne.

Fu J, Xie W, Zhou J, Qi L (2020). A method for the simultaneous identification of anisotropic yield and hardening constitutive parameters for sheet metal forming. International Journal of Mechanical Sciences, 105756.

Gambirasio L, Rizzi E (2014). On the calibration strategies of the Johnson-Cook strength model: Discussion and applications to experimental data. Materials Science and Engineering A, 610, 370-413.

Gao CY, Zhang LC (2012). Constitutive modelling of plasticity of fcc metals under extremely high strain rates. International Journal of Plasticity, 32, 121-133.

Gao X, Faleskog J, Shih CF, Dodds RH (1998). Ductile tearing in part-through cracks: Experiments and cell-model predictions. Engineering Fracture Mechanics, 59, 761-777.

Grimsmo EL, Clausen AH, Aalberg A, Langseth M (2017). Fillet welds subjected to impact loading - An experimental study. International Journal of Impact Engineering, 108, 101-113.

Grimsmo EL, Clausen AH, Langseth M, Aalberg A (2015). An experimental study of static and dynamic behaviour of bolted end-plate joints of steel. International Journal of Impact Engineering, 85, 132-145.

Gurson AL (1977). Continuum theory of ductile rupture by void nucleation and growth: Part I-Yield criteria and flow rules for porous ductile media. Journal of Engineering Materials and Technology, 99, 2-15.

Hahn GT, Kanninen MF, Rosenfield AR (1972). Fracture toughness of materials. Annual Review of Materials Science, 2, 381-404.

Hahn GT, Rosenfield AR (1975). Metallurgical factors affecting fracture toughness of aluminum alloys. Metallurgical Transactions A, 6, 653-668.

Hancock JW, Mackenzie AC (1976). On the mechanisms of ductile failure in high-strength steels subjected to multi-axial stress-states. Journal of the Mechanics and Physics of Solids, 24, 147-160. 
Huang J, Guo Y, Qin D, Zhou Z, Li D, Li Y (2018). Influence of stress triaxiality on the failure behavior of Ti-6Al-4V alloy under a broad range of strain rates. Theoretical and Applied Fracture Mechanics, 97, 48-61.

Huh H, Ahn K, Lim JH, Kim HW, Park LJ (2014). Evaluation of dynamic hardening models for BCC, FCC, and HCP metals at a wide range of strain rates. Journal of Materials Processing Technology, 214, 1326-1340.

Johnson GR, Cook WH (1983). A constitutive model and data for metals subjected to large strains, high strain rates and high temperatures. In Proceedings of the 7 th International Symposium on Ballistics, pp. 541-547.Seventh International Symposium on Ballistics, Hague.

Johnson GR, Cook WH (1985). Fracture characteristics of three metals subjected to various strains, strain rates, temperatures and pressures. Engineering Fracture Mechanics, 21, 31-48.

Kabirian F, Khan AS, Pandey A (2014). Negative to positive strain rate sensitivity in 5xxx series aluminum alloys: Experiment and constitutive modeling. International Journal of Plasticity, 55, 232-246.

Kapoor R, Nemat-Nasser S (1998). Determination of temperature rise during high strain rate deformation. Mechanics of Materials, 27, 1-12.

Karr DG, Akçay FA (2016). A criterion for ductile fracture based on continuum modeling of energy release rates. International Journal of Fracture, 197, 201-212.

Khan AS, Liang R (1999). Behaviors of three BCC metal over a wide range of strain rates and temperatures: Experiments and modeling. International Journal of Plasticity, 15, 1089-1109.

Khan, AS, Liu H (2012a). A new approach for ductile fracture prediction on Al 2024-T351 alloy. International Journal of Plasticity, 35, 1-12.

Khan AS, Liu H (2012b). Variable strain rate sensitivity in an aluminum alloy: Response and constitutive modeling. International Journal of Plasticity, 36, 1-14.

Khan AS, Liu H (2012c). Strain rate and temperature dependent fracture criteria for isotropic and anisotropic metals. International Journal of Plasticity, 37, 1-15.

Khan AS, Liu J, Yoon JW, Nambori R (2015). Strain rate effect of high purity aluminum single crystals: Experiments and simulations. International Journal of Plasticity, 67, 39-52.

Kim JH, Kim D, Han HN, Barlat F, Lee MG (2013). Strain rate dependent tensile behavior of advanced high strength steels: Experiment and constitutive modeling. Materials Science and Engineering: A, 559, 222-231.

Kim WJ, Kim HK, Kim WY, Han SW (2008). Temperature and strain rate effect incorporated failure criteria for sheet forming of magnesium alloys. Materials Science and Engineering: A, 488, 468-474.

Landau P, Osovski S, Venkert A, Gärtnerová V, Rittel D (2016). The genesis of adiabatic shear bands. Scientific Reports 6, 37226.

Larsson R, Razanica S, Josefson BL (2016). Mesh objective continuum damage models for ductile fracture. International Journal for Numerical Methods in Engineering, 106, 840-860.

Lee WS, Lin CF, Liu, TJ (2007). Impact and fracture response of sintered 316L stainless steel subjected to high strain rate loading. Materials Characterization, 58, 363-370.

Liao D, Zhu SP, Correia JA, De Jesus AM, Berto F (2020). Recent advances on notch effects in metal fatigue: A review. Fatigue \& Fracture of Engineering Materials \& Structures, 43, 637659.

Liu YJ, Sun Q (2014). A dynamic ductile fracture model on the effects of pressure, Lode angle and strain rate. Materials Science and Engineering: A, 589, 262-270. 
Longère P (2018). Adiabatic shear banding assisted dynamic failure: Some modeling issues. Mechanics of Materials, 116, 49-66.

Longère P, Dragon A (2015). Dynamic vs. quasi-static shear failure of high strength metallic alloys: Experimental issues. Mechanics of Materials, 80, 203-218.

Majzoobi GH, Mahmoudi AH, Moradi S (2016). Ductile to brittle failure transition of HSLA100 steel at high strain rates and subzero temperatures. Engineering Fracture Mechanics, 158, 179-193.

Mohr D, Marcadet SJ (2015). Micromechanically-motivated phenomenological HosfordCoulomb model for predicting ductile fracture initiation at low stress triaxialities. International Journal of Solids and Structures, 67, 40-55.

Molinari A, Mercier S, Jacques N (2014). Dynamic failure of ductile materials. Procedia IUTAM, 10, 201-220.

Molinari A, Ravichandran G (2005). Constitutive modeling of high-strain-rate deformation in metals based on the evolution of an effective microstructural length. Mechanics of Materials, 37, 737-752.

Moody NR, Garrison WM, Smugeresky JE, Costa JE (1993). The role of inclusion and pore content on the fracture toughness of powder-processed blended elemental titanium alloys. Metallurgical Transactions A, 24, 161-174.

Nahshon K, Hutchinson JW (2008). Modification of the Gurson model for shear failure. European Journal of Mechanics-A/Solids, 27, 1-17.

Needleman A, Tvergaard V (1991). An analysis of dynamic, ductile crack growth in a double edge cracked specimen. International Journal of Fracture, 49, 41-67.

Nguyen CT, Oterkus S, Oterkus E (2020). An energy-based peridynamic model for fatigue cracking. Engineering Fracture Mechanics, 241, 107373.

Nielsen KL, Tvergaard V (2010). Ductile shear failure or plug failure of spot welds modelled by modified Gurson model. Engineering Fracture Mechanics, 77, 1031-1047.

Osovski S, Srivastava A, Ponson L, Bouchaud E, Tvergaard V, Ravi-Chandar K, Needleman A (2015). The effect of loading rate on ductile fracture toughness and fracture surface roughness. Journal of the Mechanics and Physics of Solids, 76, 20-46.

Owen DM, Zhuang S, Rosakis AJ, Ravichandran G (1998). Experimental determination of dynamic crack initiation and propagation fracture toughness in thin aluminum sheets. International Journal of Fracture, 90, 153-174.

Özel T, Karpat Y (2007). Identification of constitutive material model parameters for highstrain rate metal cutting conditions using evolutionary computational algorithms. Materials and Manufacturing Processes, 22, 659-667.

Pan H, Liu J, Choi Y, Xu C, Bai Y, Atkins T (2016). Zones of material separation in simulations of cutting. International Journal of Mechanical Sciences, 115, 262-279.

Piao M, Huh H, Lee I, Ahn K, Kim H, Park, L. (2016). Characterization of flow stress at ultrahigh strain rates by proper extrapolation with Taylor impact tests. International Journal of Impact Engineering, 91, 142-157.

Placidi L, Barchiesi E, Misra A (2018a). A strain gradient variational approach to damage: A comparison with damage gradient models and numerical results. Mathematics and Mechanics of Complex Systems, 6, 77-100.

Placidi L, Misra A, Barchiesi E (2018b). Two-dimensional strain gradient damage modeling: A variational approach. Zeitschrift für angewandte Mathematik und Physik, 69, 56. 
Placidi L, Misra A, Barchiesi E (2019). Simulation results for damage with evolving microstructure and growing strain gradient moduli. Continuum Mechanics and Thermodynamics, 31, 1143-1163.

Rittel D, Weisbrod G (2001). Dynamic fracture of tungsten base heavy alloys. International Journal of Fracture, 112, 87-98.

Roth CC, Mohr D (2014). Effect of strain rate on ductile fracture initiation in advanced high strength steel sheets: Experiments and modeling. International Journal of Plasticity, 56, 19-44.

Rusinek A, Rodríguez-Martínez JA (2009). Thermo-viscoplastic constitutive relation for aluminium alloys, modeling of negative strain rate sensitivity and viscous drag effects. Materials \& Design, 30, 4377-4390.

Shahba A, Ghosh S (2016). Crystal plasticity FE modeling of Ti alloys for a range of strainrates. Part I: A unified constitutive model and flow rule. International Journal of Plasticity, 87, 48-68.

Shojaei A, Voyiadjis GZ, Tan PJ (2013). Viscoplastic constitutive theory for brittle to ductile damage in polycrystalline materials under dynamic loading. International Journal of Plasticity, 48, 125-151.

Siddiq A (2019). A porous crystal plasticity constitutive model for ductile deformation and failure in porous single crystals. International Journal of Damage Mechanics, 28, 233-248.

Siddiq A, Arciniega R, El Sayed T (2012). A variational void coalescence model for ductile metals. Computational Mechanics, 49, 185-195.

Siddiq A, Schmauder S (2005). Simulation of hardening in high purity niobium single crystals during deformation. Steel Grips: Journal of Steel and Related Materials, 3, 281-286.

Sjöberg T, Kajberg J, Oldenburg M (2017). Fracture behaviour of Alloy 718 at high strain rates, elevated temperatures, and various stress triaxialities. Engineering Fracture Mechanics, 178, 231-242.

Smerd R, Winkler S, Salisbury C, Worswick M, Lloyd D, Finn M (2005). High strain rate tensile testing of automotive aluminum alloy sheet. International Journal of Impact Engineering, 32, 541-560.

Spagnuolo M, Barcz K, Pfaff A, Dell'Isola F, Franciosi P (2017). Qualitative pivot damage analysis in aluminum printed pantographic sheets: numerics and experiments. Mechanics Research Communications, 83, 47-52.

Srivastava A, Ponson L, Osovski S, Bouchaud E, Tvergaard V, Needleman A (2014). Effect of inclusion density on ductile fracture toughness and roughness. Journal of the Mechanics and Physics of Solids, 63, 62-79.

Sung JH, Kim JH, Wagoner RH (2010). A plastic constitutive equation incorporating strain, strain-rate, and temperature. International Journal of Plasticity, 26, 1746-1771.

Tanimura S, Tsuda T, Abe A, Hayashi H, Jones N (2014). Comparison of rate-dependent constitutive models with experimental data. International Journal of Impact Engineering, 69, 104-113.

Tvergaard V (1981). Influence of voids on shear band instabilities under plane strain conditions. International Journal of Fracture, 17, 389-407.

Ulacia I, Salisbury CP, Hurtado I, Worswick MJ (2011). Tensile characterization and constitutive modeling of AZ31B magnesium alloy sheet over wide range of strain rates and temperatures. Journal of Materials Processing Technology, 211, 830-839.

Vaz-Romero A, Rodríguez-Martínez JA, Arias A (2015). The deterministic nature of the fracture location in the dynamic tensile testing of steel sheets. International Journal of Impact Engineering, 86, 318-335. 
Voyiadjis GZ, Abed FH (2005a). Effect of dislocation density evolution on the thermomechanical response of metals with different crystal structures at low and high strain rates and temperatures. Archives of Mechanics, 57, 299-343.

Voyiadjis GZ, Abed FH (2005b). Microstructural based models for bcc and fcc metals with temperature and strain rate dependency. Mechanics of Materials, 37, 355-378.

Wang B, Xiao X, Astakhov VP, Liu Z (2019). The effects of stress triaxiality and strain rate on the fracture strain of Ti6A14V. Engineering Fracture Mechanics, 219, 106627.

Wierzbicki T, Bao Y, Lee YW, Bai Y (2005). Calibration and evaluation of seven fracture models. International Journal of Mechanical Sciences, 47, 719-743.

Xia L, Shih CF (1995). Ductile crack growth-I. A numerical study using computational cells with microstructurally-based length scales. Journal of the Mechanics and Physics of Solids, 43, 233-259.

Yu X, Li L, Li T, Qin D, Liu S, Li Y (2017). Improvement on dynamic fracture properties of magnesium alloy AZ31B through equal channel angular pressing. Engineering Fracture Mechanics, 181, 87-100.

Yu T, Hyer H, Sohn Y, Bai Y, Wu D (2019). Structure-property relationship in high strength and lightweight AlSi10Mg microlattices fabricated by selective laser melting. Materials \& Design, 182, 108062.

Zener C, Hollomon JH (1944). Effect of strain rate upon plastic flow of steel. Journal of Applied Physics, 15, 22-32.

Zerilli FJ, Armstrong RW (1987). Dislocation-mechanics-based constitutive relations for material dynamics calculations. Journal of Applied Physics, 61, 1816-1825.

Zhang C, Voyiadjis GZ (2016). Rate-dependent size effects and material length scales in nanoindentation near the grain boundary for a bicrystal FCC metal. Materials Science and Engineering: A, 659, 55-62.

Zheng C, Wang F, Cheng X, Liu J, Fu K, Liu T, Zhu Z, Yang K, Peng M, Jin D (2015). Failure mechanisms in ballistic performance of $\mathrm{Ti}-6 \mathrm{Al}-4 \mathrm{~V}$ targets having equiaxed and lamellar microstructures. International Journal of Impact Engineering, 85, 161-169. 